\title{
The adenosine triphosphate-sensitive potassium channel opener nicorandil protects the ischemic rabbit spinal cord
}

\author{
Yutaka Wakamatsu, MD \\ Norihiko Shiiya, MDa \\ Takashi Kunihara, MDa \\ Satoshi Watanabe, $\mathrm{MD}^{\mathrm{b}}$ \\ Keishu Yasuda, MDa
}

Objective: We investigated the protective effects of an adenosine triphosphate-sensitive potassium channel opener nicorandil in the rabbit model of spinal cord ischemia.

Methods: Rabbits were randomized into 4 groups (each $n=6$ ): the nicorandil group (100 $\mu \mathrm{g} / \mathrm{kg}$ intravenous nicorandil 10 minutes before ischemia); the glibenclamide plus nicorandil group ( $3 \mathrm{mg} / \mathrm{kg}$ intravenous glibenclamide, an antagonist of adenosine triphosphate-sensitive potassium channels, 10 minutes before nicorandil administration); the vehicle group (vehicle alone); and the sham operation group (without spinal cord ischemia). Spinal cord ischemia was induced by balloon occlusion of the infrarenal abdominal aorta for 15 minutes at $39^{\circ} \mathrm{C}$. Neurologic function was graded into Johnson's score at 8 hours, 1 day, and 2 days. Histopathologic examination was performed at 2 days, and the number of intact motor neuron cells was compared.

Results: Johnson scores of the glibenclamide plus nicorandil and vehicle groups were significantly lower than those of the sham operation and nicorandil groups at each time point, and no statistically significant difference was observed between the glibenclamide plus nicorandil and vehicle groups. Histopathologic examination revealed that motor neurons were almost normal in the nicorandil group, whereas about $55 \%$ of motor neurons were lost in the vehicle and glibenclamide plus nicorandil groups.

Conclusions: Nicorandil has a protective effect on the ischemic rabbit spinal cord, and the beneficial effect seems mediated through the activation of adenosine triphosphate-sensitive potassium channels.

Surgery a and Department of Anatomy, Hokkaido University, Sapporo, Japan.

Received for publication Dec 1, 2000; revisions requested Feb 16, 2001; revisions received Feb 28, 2001; accepted for publication March 9, 2001.

Address for reprints: Norihiko Shiiya, MD, Department of Cardiovascular Surgery, Hokkaido University Hospital, N14W5, kita-ku, Sapporo 060-8648, Japan (E-mail: shiyanor@med.hokudai.ac.jp).

J Thorac Cardiovasc Surg 2001;122:728-33

Copyright (C) 2001 by The American Association for Thoracic Surgery $0022-5223 / 2001 \$ 35.00+0 \quad \mathbf{1 2 / 1 / 1 1 5 7 0 3}$

doi: $10.1067 / \mathrm{mtc} .2001 .115703$

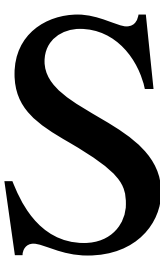

pinal cord injury remains a serious problem after operations on the descending and thoracoabdominal aorta. A number of techniques and drugs that may reduce the ischemia-reperfusion injury of the cord have been evaluated both clinically and experimentally, with variable success.

The adenosine triphosphate-sensitive potassium channels (KATPs) have been shown to be involved in the mechanisms of ischemic preconditioning in the heart ${ }^{1}$ and in the brain, ${ }^{2}$ and KATP openers may thus have protective effects on these organs. In the brain neuronal cells, protective effects of KATP openers, which are blocked by their inhibitors, have been reported. ${ }^{3,4}$ In the spinal cord, however, there is no report that examines the protective effects of KATP openers. The present study 
TABLE 1. Arterial blood gas and glucose level before ischemia

\begin{tabular}{lccccc}
\hline Group & $\mathbf{p H}$ & $\mathbf{P o}_{\mathbf{2}}(\mathbf{m m ~ H g})$ & $\mathbf{P c o}_{2}(\mathbf{m m ~ H g})$ & Base excess & $\mathbf{G l u c o s e}(\mathbf{m g} / \mathbf{d L})$ \\
\hline Sham operation & $7.36 \pm 0.01$ & $436 \pm 76$ & $39.3 \pm 2.8$ & $-2.8 \pm 0.7$ & $115 \pm 9$ \\
Vehicle & $7.38 \pm 0.05$ & $443 \pm 35$ & $38.6 \pm 1.7$ & $-1.7 \pm 1.1$ & $110 \pm 5$ \\
Nicorandil & $7.35 \pm 0.05$ & $414 \pm 55$ & $40.9 \pm 2.9$ & $-2.5 \pm 0.8$ & $136 \pm 6.7$ \\
G+N & $7.39 \pm 0.05$ & $422 \pm 25$ & $36.3 \pm 1.6$ & $-2.7 \pm 1.0$ & $117 \pm 17$
\end{tabular}

was undertaken to examine whether intravenous administration of nicorandil, a clinically available KATP opener, protects the spinal cord motor neurons and motor function after reperfusion.

\section{Material and Methods}

Twenty-four male New Zealand White rabbits weighing 2.5 to 3.5 $\mathrm{kg}$ were used in this study. The animal protocol was approved by the Hokkaido University Animal Care and Use Committee and was in accordance with the "Guide for the Care and Use of Laboratory Animals" published by the National Academy Press (revised 1996). All experiments were performed in the Research Institute of Hokkaido University School of Medicine.

\section{Surgical Procedure}

The animals were anesthetized with intramuscular administration of ketamine hydrochloride $(50 \mathrm{mg} / \mathrm{kg}$ ) and maintained with $2 \%$ halothane in $100 \%$ oxygen. They were allowed to breathe spontaneously with a nonsealing face-mask device. A rectal probe was inserted to monitor body temperature, which was maintained at $39^{\circ} \mathrm{C}$ (normal value in our animals) with the aid of a heating pad (Asahi Plate Warmer TK-43; Asahi Denshi Inc, Osaka, Japan). The marginal ear vein was cannulated for intravenous drug administration. The median ear artery was cannulated to obtain arterial blood samples and to monitor proximal aortic blood pressure. Arterial blood gas and glucose levels were measured after anesthetic stabilization. Heart rate from the electrocardiogram and arterial blood pressure were continuously monitored and recorded throughout the operation.

After intravenous administration of heparin sodium (100 $\mathrm{IU} / \mathrm{kg}$ ), a $4 \mathrm{~F}$ angiographic balloon catheter (A024; Goodtec, Huntington Beach, Calif) was introduced through the surgically isolated left femoral artery into the abdominal aorta to a distance of $15 \mathrm{~cm}$ from the arteriotomy. This distance was decided on so that the tip of the catheter balloon lay just caudal to the left renal artery and was confirmed at postmortem examination of the rabbits killed for catheter placement measurements. Spinal cord ischemia was induced by inflation of the balloon. Aortic occlusion was confirmed by reduction in distal aortic blood pressure, which was measured through the side hole of the balloon catheter. At the end of the operation, catheters were removed, surgical wounds were closed, and the rabbits were allowed to recover at ambient temperature and were returned to their cages.

\section{Experimental Protocol}

The rabbits were randomized into 4 groups. In the nicorandil group ( $\mathrm{n}=6$ ), $100 \mu \mathrm{g} / \mathrm{kg}$ nicorandil (Chugai Pharmaceutical, Tokyo,

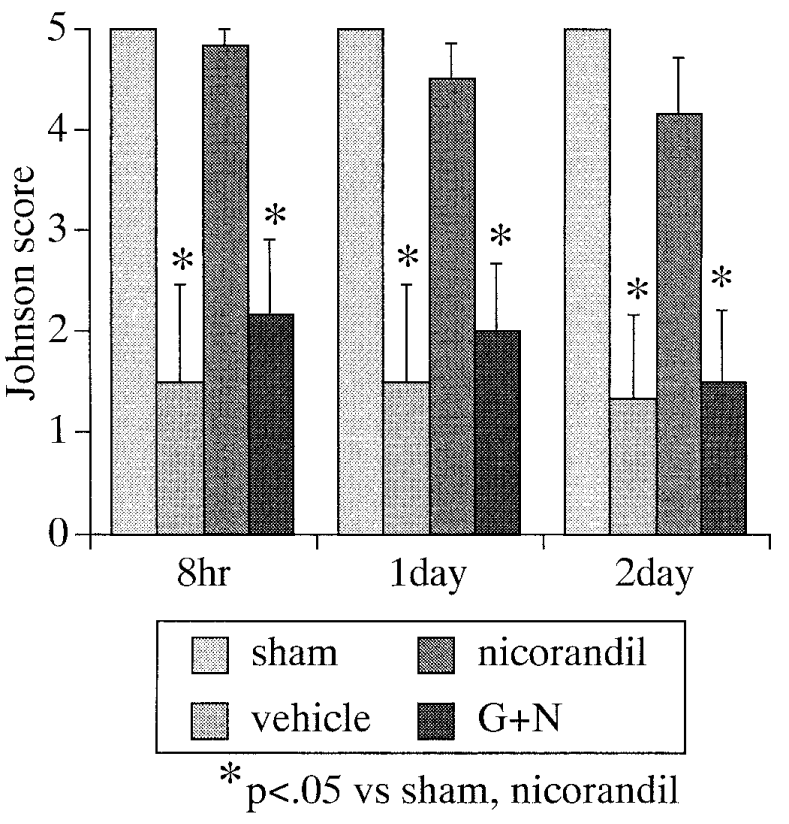

Figure 1. Neurologic outcome expressed by using the Johnson 5point scale.

Japan) was intravenously administered 10 minutes before the rabbits underwent 15 minutes of spinal cord ischemia. The 15-minute ischemic insult and the dosage schedule of nicorandil were based on results of preliminary experimentation. Arterial blood samples were collected to measure plasma concentrations of nicorandil, which were undetectable before administration of the drug and $40.7 \pm 4.5 \mathrm{ng} / \mathrm{mL}$ at the onset of ischemia. Nicorandil concentrations 10 minutes after reperfusion were $6.2 \mathrm{ng} / \mathrm{mL}$ in 2 animals and undetectable (the minimum detectable level was $5 \mathrm{ng} / \mathrm{mL}$ ) in the rest. High-performance liquid chromatography ${ }^{5}$ was used to determine plasma concentration.

In the glibenclamide plus nicorandil $(\mathrm{G}+\mathrm{N})$ group $(\mathrm{n}=6), 3$ $\mathrm{mg} / \mathrm{kg}$ glibenclamide (Wako Pure Chemical Industries, Osaka, Japan), a blocker of KATPs, was intravenously administered 20 minutes before ischemia, and the animals had a similar time of ischemia and the same dosage schedule of nicorandil. Glibenclamide was dissolved in $0.1 \mathrm{~N} \mathrm{NaOH}$, and $\mathrm{pH}$ was adjusted to 7.6 with $0.1 \mathrm{~N} \mathrm{HCl}^{6}$ Rabbits in the vehicle group $(\mathrm{n}=6)$ had a similar time of ischemia but received the same volume of saline solution instead of nicorandil. Rabbits in the sham operation group $(n=6)$ underwent similar instrumentation and received the same volume of saline solution without spinal cord ischemia. 

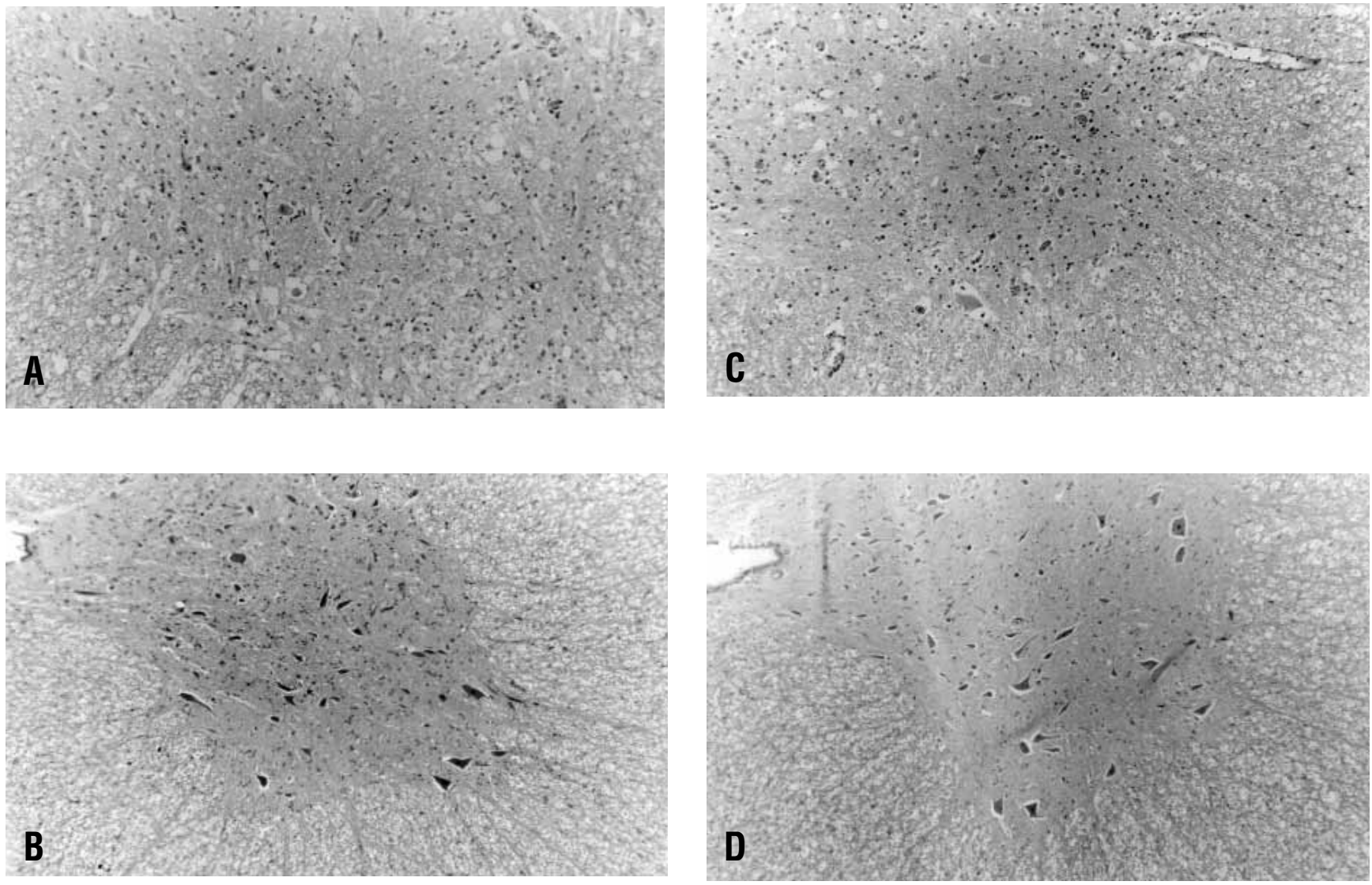

Figure 2. Photomicrographs of the spinal cord stained with hematoxylin and eosin: A, sham operation group; B, vehicle group; $C$, nicorandil group; and $D, G+N$ group. (Original magnification, $100 \times$.)

\section{Neurologic Assessment}

Neurologic function was evaluated at 8 hours, 1 day, and 2 days after operation. Animal neurologic function was graded on a 5point scale according to the method of Johnson and colleagues ${ }^{7}$ : 0 , hind-limb paralysis; 1 , severe paraparesis; 2 , functional movement but no hop; 3 , ataxia with disconjugate hop; 4, minimal ataxia; and 5 , normal function. One member of the research team without knowledge of the treatment graded neurologic function.

\section{Histopathology}

Immediately after neurologic testing at 2 days, the animals were killed after achievement of deep anesthesia with sodium pentobarbital (50 mg/kg administered intravenously), and the spinal cords were quickly removed. They were fixed by immersion in $2 \%$ paraformaldehyde- $15 \%$ picric acid in $0.1 \mathrm{~mol} / \mathrm{L}$ phosphate buffer and embedded in paraffin. Serial transverse sections $(5 \mu \mathrm{m})$ were obtained at the L4-L5 level. They were stained with hematoxylin and eosin for histopathologic observations. In 4 serial sections of each rabbit, the total number of motor neurons in the gray matter was counted and averaged.

\section{Statistical Analysis}

Data are expressed as means \pm standard error of the mean. MannWhitney rank sum tests were used for nonparametric comparisons of the Johnson score between 2 groups. One-way analysis of variance and Fisher post hoc testing was used for comparison of continuous variables.

\section{Results}

Arterial blood gas and glucose levels before ischemia were not different among the 4 groups (Table 1). Heart rates, mean proximal and distal aortic pressures, and rectal temperatures at baseline (before drug treatment), immediately before aortic occlusion, during aortic occlusion, and $30 \mathrm{~min}-$ utes after reperfusion are shown in Table 2. Heart rates and mean proximal aortic pressures were not different among the 4 groups at each time point. In the nicorandil group, bolus infusion of nicorandil resulted in a transient but significant decrease of mean proximal aortic pressure to $72.5 \pm$ $3.4 \mathrm{~mm} \mathrm{Hg}$ at 5 minutes after drug treatment $(P=.014$ from baseline, paired $t$ test), which returned to baseline before aortic occlusion. By contrast, mean proximal aortic pressure did not show a significant decrease in the $\mathrm{G}+\mathrm{N}$ group $(72.2$ $\pm 4.9 \mathrm{~mm} \mathrm{Hg}$ at 5 minutes after drug treatment, $P=.1167$ from baseline, paired $t$ test). Heart rate remained unchanged in both groups. In the 3 groups with spinal cord ischemia, mean distal aortic pressure decreased to less than $20 \mathrm{~mm} \mathrm{Hg}$ 
TABLE 2. Heart rate, mean proximal and distal aortic pressure, and rectal temperature

\begin{tabular}{|c|c|c|c|c|}
\hline & Heart rate (beats/min) & Distal MAP (mm Hg) & Proximal MAP (mm Hg) & Rectal temperature $\left({ }^{\circ} \mathrm{C}\right)$ \\
\hline \multicolumn{5}{|c|}{ Baseline (before drug treatment) } \\
\hline Sham group & $296 \pm 10$ & $82.3 \pm 2.3$ & $77.5 \pm 4.7$ & $39.4 \pm 0.1$ \\
\hline Vehicle group & $305 \pm 14$ & $85.0 \pm 3.9$ & $77.4 \pm 4.5$ & $39.4 \pm 0.4$ \\
\hline Nicorandil group & $296 \pm 18$ & $87.7 \pm 2.8$ & $79.3 \pm 2.8$ & $39.5 \pm 0.3$ \\
\hline $\mathrm{G}+\mathrm{N}$ group & $310 \pm 17$ & $81.3 \pm 4.5$ & $76.0 \pm 6.1$ & $39.5 \pm 0.2$ \\
\hline \multicolumn{5}{|c|}{ Immediately before aortic occlusion } \\
\hline Sham group & $299 \pm 9$ & $82.7 \pm 1.4$ & $77.0 \pm 2.1$ & $39.4 \pm 0.1$ \\
\hline Vehicle group & $306 \pm 15$ & $82.7 \pm 3.4$ & $75.8 \pm 3.5$ & $39.3 \pm 0.4$ \\
\hline Nicorandil group & $301 \pm 19$ & $84.0 \pm 3.0$ & $74.2 \pm 2.4$ & $39.2 \pm 0.3$ \\
\hline $\mathrm{G}+\mathrm{N}$ group & $288 \pm 23$ & $85.2 \pm 2.9$ & $77.2 \pm 5.5$ & $39.2 \pm 0.2$ \\
\hline \multicolumn{5}{|c|}{ During aortic occlusion (10 min) } \\
\hline Sham group & $296 \pm 7$ & $82.7 \pm 1.0$ & $77.7 \pm 1.7$ & $39.4 \pm 0.1$ \\
\hline Vehicle group & $302 \pm 15$ & $18.0 \pm 2.4^{*}$ & $76.0 \pm 5.2$ & $38.6 \pm 0.3$ \\
\hline Nicorandil group & $265 \pm 26$ & $17.5 \pm 1.0^{*}$ & $77.3 \pm 1.8$ & $38.7 \pm 0.2$ \\
\hline $\mathrm{G}+\mathrm{N}$ group & $242 \pm 18$ & $14.3 \pm 1.0^{*}$ & $78.0 \pm 3.8$ & $38.7 \pm 0.3$ \\
\hline \multicolumn{5}{|c|}{30 min after reperfusion } \\
\hline Sham group & $297 \pm 7$ & $81.0 \pm 4.5$ & $78.3 \pm 2.2$ & $39.4 \pm 0.1$ \\
\hline Vehicle group & $314 \pm 14$ & $80.7 \pm 4.5$ & $75.4 \pm 6.0$ & $39.2 \pm 0.3$ \\
\hline Nicorandil group & $278 \pm 24$ & $83.7 \pm 2.7$ & $76.2 \pm 0.9$ & $39.1 \pm 0.3$ \\
\hline $\mathrm{G}+\mathrm{N}$ group & $283 \pm 15$ & $80.7 \pm 2.5$ & $76.0 \pm 1.1$ & $39.1 \pm 0.2$ \\
\hline
\end{tabular}

$M A P$, Mean aortic pressure.

${ }^{*} P<.05$ versus sham operation group.

after balloon inflation, and no pulsation was recorded. The rectal temperature decreased by $1^{\circ} \mathrm{C}$ on average during balloon inflation and returned to normal level within $10 \mathrm{~min}$ utes after reperfusion. No significant difference was found in these parameters among the 3 groups at each time point.

\section{Neurologic Outcome}

Neurologic outcome data are shown in Figure 1. All rabbits in the sham operation group were normal (grade 5) throughout the observation period. In the nicorandil group all but 1 of the rabbits had normal neurologic function 8 hours after operation, and 2 rabbits, including the one with injury at 8 hours, showed functional deterioration 1 and 2 days after operation. There was no statistically significant difference between the 2 groups. By contrast, the scores of the $\mathrm{G}+\mathrm{N}$ and vehicle groups were significantly lower than those of the sham operation and nicorandil groups at each time point, and no statistically significant difference was observed between the $\mathrm{G}+\mathrm{N}$ and vehicle groups.

\section{Histopathology}

Histopathology data are shown in Figure 2. In the spinal cord anterior horn of the rabbits in the sham operation group and those in the nicorandil group, except the 2 with functional deterioration, large motor neurons showed no histologic change. In the vehicle and $\mathrm{G}+\mathrm{N}$ groups, however, the number of intact motor neurons decreased, and the remaining motor neurons showed shrinkage and chromatic agglu- tination. The total number of motor neurons in the gray matter was significantly different among the 4 groups (Figure 3 ). It was significantly lower in the vehicle and $\mathrm{G}+\mathrm{N}$ groups than that in the sham operation and nicorandil groups, and there was no significant difference between the vehicle and $\mathrm{G}+\mathrm{N}$ groups and between the sham operation and nicorandil groups. This result shows good correlation with the Johnson score at 2 days before animal death.

\section{Discussion}

The results of the present study showed that intravenous administration of nicorandil before ischemia had a protective effect on the rabbit spinal cord motor neurons and motor function, and the beneficial effect was lost by glibenclamide pretreatment. The number of motor neurons was parallel to the motor function score. Fifteen minutes' occlusion of infrarenal abdominal aorta in the rabbit has been used by Sakurai and colleagues ${ }^{8}$ as a model of spinal cord ischemia. They have reported that at $37^{\circ} \mathrm{C}, 40 \%$ of rabbits were neurologically intact 2 days after the operation, but no rabbit had normal neurologic function at 7 days. In our study, however, no rabbit had normal neurologic function at 2 days, and this difference may be explained by the higher temperature used in our study. Suzuki and colleagues ${ }^{6}$ have reported that intravenous administration of the KATP openers nicorandil and pinacidil accelerates the recovery of reflex potential after spinal cord ischemia in cats ${ }^{6}$ but did not evaluate the motor function. To our knowledge, the present study is the first to 
cell count / slice

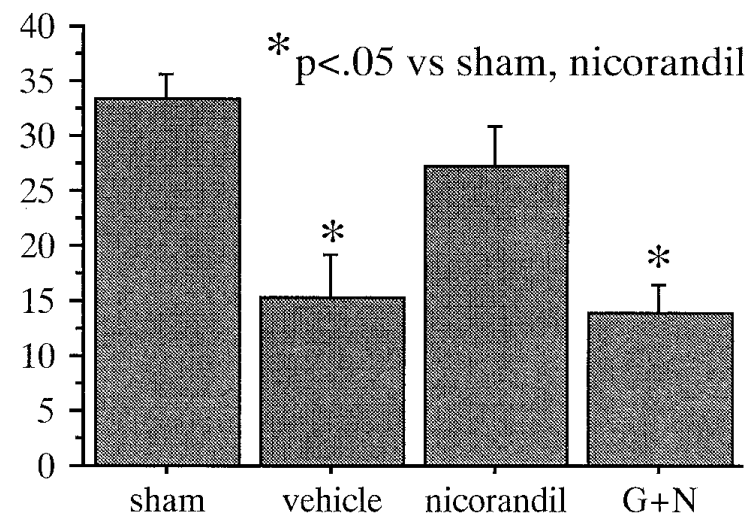

Figure 3. Total number of motor neurons in the gray matter.

demonstrate the protective effect of a KATP opener on motor function in the model of spinal cord ischemia.

Nicorandil is a hybrid drug exerting a dual mechanism of action as a KATP opener and a nitrate. ${ }^{9}$ It has been reported that KATP activation is responsible for the vasodilatory effects on the resistance vessels, whereas the nitrate action is predominant in the conductance vessels. ${ }^{10}$ Therefore these vasodilatory effects or a direct effect on neuronal KATPs may have contributed to its protective effect on the ischemic spinal cord. The nitrate action, however, does not seem involved because the protective effect of nicorandil was completely blocked by glibenclamide. Suzuki and colleagues ${ }^{6}$ have shown, in the cat model of spinal cord ischemia, that $3 \mathrm{mg} / \mathrm{kg}$ glibenclamide completely blocks the beneficial effect of 100 $\mu \mathrm{g} / \mathrm{kg}$ nicorandil but does not have a detrimental effect if administered alone. Therefore, the protective effects of nicorandil seem mediated through activation of KATP.

The dosage schedule of nicorandil in the present study was carefully decided so that the therapeutic plasma concentration could be obtained at the onset of ischemia but not during the reperfusion period. Consequently, the plasma concentration of the drug at the onset of ischemia in this study roughly corresponded to that after a single oral dose of $5 \mathrm{mg}$ in the human subject. ${ }^{11}$ This was based on the following considerations. Nitrates and other nitric oxide (NO) donors have been reported to be harmful rather than protective during spinal cord ischemia, despite their vasodilatory effects. ${ }^{12}$ In the brain it has been reported that protective vascular effects of endothelial NO outweigh the neurotoxic potential of neuronal NO only in the very early stages of ischemia, and large amounts of NO produced by inducible NO synthase during the late reperfusion period contribute to the delayed progression of the damage. ${ }^{13}$ In the heart Ohno and colleagues ${ }^{14}$ have reported that nicorandil has a protective effect if it is administered before ischemia but not if it is administered after ischemia, and this effect is mediated through the activation of KATP.
The specific mechanism underlying the beneficial effect of a KATP opener on the ischemic spinal cord remains to be clarified. The vasodilatory effect through vascular KATP activation, hyperpolarization of the neurons through sarcolemnal KATP activation, ${ }^{3,4}$ and ischemic tolerance afforded by mitochondrial KATP activation may have been involved. Because nicorandil at this concentration does not seem to dilate the resistance vessels through KATP activation, ${ }^{15}$ and both proximal and distal aortic pressures were not different at the moment of spinal cord ischemia among the 3 groups, we believe that there was very little contribution of the vasodilatory effect. Although we did not measure the regional spinal cord blood flow, Suzuki and colleagues ${ }^{6}$ have reported that it does not show an increase by administration of 30 to 100 $\mu \mathrm{g} / \mathrm{kg}$ nicorandil in the cat model of spinal cord ischemia. In the heart it has been reported that ischemic tolerance afforded by KATP activation is mediated through the mitochondrial, rather than the sarcolemmal, KATP. ${ }^{1}$ Sato and colleagues ${ }^{16}$ have reported that nicorandil is 100 times more potent in opening mitochondrial KATPs than in opening sarcolemnal KATP in the rabbit myocytes. A recent study with diazoxide and 5-hydroxydecanoic acid, a selective mitochondrial KATP opener and blocker, has suggested involvement of mitochondrial KATPs in the protective effect of KATP openers in the brain. ${ }^{17}$ Therefore, we suppose that the protective effect of nicorandil on the spinal cord is mediated through the mitochondrial KATP. Further research works with selective mitochondrial KATP openers will be required on this issue.

In the present study we used ketamine and halothane as anesthetics. Although some volatile anesthetics have been reported to have cardioprotective effects through their action on KATPs, halothane has been shown to have neither this effect ${ }^{18}$ nor a protective effect on the spinal cord. ${ }^{19}$ Ketamine, on the other hand, is a noncompetitive $N$-methyl-D-aspartate antagonist and has been reported to have a protective effect on the spinal cord. ${ }^{19}$ Therefore, the result of the present study may have been influenced by the effect of ketamine.

In conclusion, results of the present study show that nicorandil has a protective effect on the ischemic rabbit spinal cord, and the beneficial effect seems mediated through the activation of KATPs.

\section{References}

1. Grover GJ, Garlid KD. ATP-sensitive potassium channels: a review of their cardioprotective pharmacology. J Mol Cell Cardiol. 2000;32:677-95.

2. Heurteaux C, Lauritzen I, Widmann C, Lazdunski M. Essential role of adenosine, adenosine A1 receptors, and ATP-sensitive $\mathrm{K}+$ channels in cerebral ischemic preconditioning. Proc Natl Acad Sci U S A. 1995;92: 4666-70.

3. Heurteaux C, Bertaina V, Widmann C, Lazdunski M. $\mathrm{K}^{+}$channel openers prevent global ischemia-induced expression of $\mathrm{c}$-fos, $\mathrm{c}$-jun, heat shock protein, and amyloid beta-protein precursor genes and neuronal death in rat hippocampus. Proc Natl Acad Sci U S A. 1993;90:9431-5.

4. Lauritzen I, De Weille JR, Lazdunski M. The potassium channel opener (-) - cromakalim prevents glutamate-induced cell death in hippocampal neurons. J Neurochem. 1997;69:1570-9.

5. Tanikawa M, Uzu M, Ohsawa Y, Fukushima M. Sensitive method for 
determination of nicorandil in human plasma by reversed-phase highperformance liquid chromatography with ultraviolet detection. $J$ Chromatogr. 1993;617:163-7.

6. Suzuki T, Sekikawa T, Nemoto T, Moriya H, Nakaya H. Effects of nicorandil on the recovery of reflex potentials after spinal cord ischaemia in cats. Br J Pharmacol. 1995;116:1815-20.

7. Johnson SH, Kraimer JM, Graeber GM. Effects of flunarizine on neurological recovery and spinal cord blood flow in experimental spinal cord ischemia in rabbits. Stroke. 1993;24:1547-53.

8. Sakurai M, Hayashi T, Abe K, Aoki M, Sadahiro M, Tabayashi K. Enhancement of heat shock protein expression after transient ischemia in the preconditioned spinal cord of rabbits. J Vasc Surg. 1998;27:720-5.

9. Taira N. Nicorandil as a hybrid between nitrates and potassium channel activators. Am J Cardiol. 1989;63:18J-24J.

10. Sakai K, Akima M, Kamachi S, Moriyasu M, Kitajima S. Interrelationship of cardiovascular effects, plasma levels of nicorandil, and vascular cGMP formation in conscious rats. J Pharm Pharmacol. 1998;50:661-6.

11. Frydman AM, Chapelle P, Diekmann H, et al. Pharmacokinetics of nicorandil. Am J Cardiol. 1989;63:25J-33J.

12. Simpson JI, Eide TR, Schiff GA, et al. Effect of nitroglycerin on spinal cord ischemia after thoracic aortic cross-clamping. Ann Thorac Surg. 1996;61:113-7.
13. Iadecola C. Bright and dark sides of nitric oxide in ischemic brain injury. Trends Neurosci. 1997;20:132-9.

14. Ohno Y, Minatoguchi S, Uno Y, et al. Nicorandil reduces myocardial infarct size by opening the K(ATP) channel in rabbits. Int J Cardiol. 1997;62:181-90.

15. Nakae I, Quan L, Hashimoto K, Sugimoto Y, Tsutamoto T, Kinoshita M. Mechanism of the vasodilatory action of nicorandil on coronary circulation in dogs. Cardiovasc Drugs Ther. 1994;8:137-45.

16. Sato T, Sasaki N, O'Rourke B, Marban E. Nicorandil, a potent cardioprotective agent, acts by opening mitochondrial ATP-dependent potassium channels. J Am Coll Cardiol. 2000;35:514-8.

17. Domoki F, Perciaccante JV, Veltkamp R, Bari F, Busija DW. Mitochondrial potassium channel opener diazoxide preserves neuronal-vascular function after cerebral ischemia in newborn pigs. Stroke. 1999;30:2713-9.

18. Roscoe AK, Christensen JD, Lynch C. Isoflurane, but not halothane, induces protection of human myocardium via adenosine A1 receptors and adenosine triphosphate-sensitive potassium channels. Anesthesiology. 2000;92:1692-701.

19. Naslund TC, Hollier LH, Money SR, Facundus EC, Skenderis B. Protecting the ischemic spinal cord during aortic clamping: the influence of anesthetics and hypothermia. Ann Surg. 1992;215: $409-15$.

The Journal of Thoracic and Cardiovascular Surgery delivers the information you need now. Articles usually appear within four months of acceptance. 